\title{
Bases para um Novo Sanitarismo
}

\section{Basis for health care reform}

\section{Bases para la reforma del sistema de salud}

Gustavo Diniz Ferreira Gusso. Pesquisador independente. São Paulo, SP, Brasil. gustavo.gusso@usp.br

(Autor correspondente)

Daniel Knupp. Pesquisador independente. Belo Horizonte, MG, Brasil. knupp.bh@gmail.com

Thiago Gomes da Trindade. Pesquisador independente. Natal, RN, Brasil. thiagogtrindade@gmail.com

Nulvio Lermen Junior. Pesquisador independente. Florianópolis, SC, Brasil. nulvio@ hotmail.com

Paulo Poli Neto. Pesquisador independente. Curitiba, PR, Brasil. ppolineto@gmail.com

\section{Resumo}

As reformas dos sistemas de saúde dos países da Europa Ocidental e Canadá na segunda metade do século XX inspiraram o movimento conhecido como Reforma Sanitária no Brasil. Esses países implementaram sistemas de saúde socializados integrando financiamento público e serviços privados, alguns originados da universalização do seguro-saúde (bismarckiano) outros da arrecadação geral de impostos (beveridgeano). Nesse processo, um dos fatores que mais evoluiu foi a contratualização, que permite a identificação de fraudes e promove a saúde no nível individual e coletivo em vez do uso indiscriminado dos serviços. No Brasil, a Constituição de 1988 lança as bases para um sistema universal de saúde financiado por impostos gerais que permite a ampliação de serviços privados sem regulação estatal, mas cofinanciados pelo Estado por meio da isenção fiscal e subsídios para servidores públicos. Nesse cenário, cresce a dicotomia, e a sobreposição entre serviços estatais e privados é agudizada.

\section{Abstract}

The reforms in the health systems of Western European countries and Canada in the second half of the twentieth century inspired the movement known as Health Reform in Brazil. These countries have implemented socialized health systems integrating public funding and private services, some originated from universal health insurance (Bismarckian) and others from general tax revenues (Beveridgean). In this process, one of the factors that has evolved is the contracting that enables identification of fraud and promotes health at the individual and collective level rather than indiscriminate use of services. In Brazil, the 1988 Constitution lays the foundation for a universal health care system funded by general taxes while allowing the expansion of private services without state regulation, but co-financed by the state through tax exemption and subsidies for public employees. In this scenario, dichotomy increases, and the overlap between state and private services is aggravated.

\section{Resumen}

Las reformas de los sistemas de salud de los países de Europa Occidental y Canadá en la segunda mitad del siglo XX inspiraron el movimiento conocido como la Reforma de Salud en Brasil. Estos países han puesto en práctica sistemas de salud socializados que integran la financiación pública y los servicios privados, algunos se originaron de la universalización del seguro-salud (bismarckiano), otros de la recaudación de impuestos generales (beveridgeano). En este proceso uno de los factores que ha evolucionado es la contractualización que permite la identificación de fraude y promueve la salud en el nivel individual y colectivo más que el uso indiscriminado de los servicios. En Brasil, la Constitución de 1988 pone las bases para un sistema de salud universal financiado por impuestos generales al tiempo que permite la expansión de los servicios privados y sin regulación estatal, pero cofinanciados por el Estado a través de la exención fiscal y subsidios para servidores públicos. En este escenario crece la dicotomía, y el solapamiento entre los servicios estatales y privados se agrava.

Como citar: Gusso GDF, Knupp D, Trindade TG, Lermen Junior N, Poli Neto P. Bases para um Novo Sanitarismo. Rev Bras Med Fam Comunidade. 2015;10(36):1-10.

http://dx.doi.org/10.5712/rbmfc10(36)1056
Palavras-chave:

Reforma dos Serviços de Saúde

Atenção Primária à Saúde

Sistema Único de Saúde

Keywords:

Health Care Reform

Primary Health Care

Unified Health System

Palabras clave:

Reforma de La

Atención de Salud

Atención Primaria a la Salud

Sistema Único de Salud

Fonte de financiamento:

declaram não haver.

Parecer CEP:

não se aplica.

Conflito de interesses:

declaram não haver.

Procedência e revisão por pares: revisado por pares.

Recebido em: 19/12/2014.

Aprovado em: 02/02/2015. 


\section{Introdução}

No início da década de 1970, o Brasil viu surgir um movimento, eminentemente acadêmico em sua origem, ao qual se denomina Movimento da Reforma Sanitária. ${ }^{1}$ A saúde como direito de todos e dever do Estado, conceito que se cristaliza no artigo 196 da Carta Magna, era uma das bandeiras defendidas por esse movimento. A redemocratização do país estava no centro dessa pauta que propunha uma série de reformas sociais. Os participantes desse movimento tiveram um importante papel na década de 1980, destacando-se o relatório da $8^{a}$ Conferência Nacional de Saúde, cujos apontamentos influenciaram sobremaneira na criação do Sistema Único de Saúde (SUS) quando da promulgação da Constituição Federal.

Quatro décadas se passaram desde as origens dessa reforma sanitária e o contexto político e social do país é consideravelmente diferente. O avanço no processo democrático e as conquistas sociais, entre as quais o próprio SUS, foram marcantes nesse período. Apesar disso, os princípios fundamentais do SUS ainda não são plenamente vigentes. Persistem problemas que já se tornaram crônicos, como, por exemplo, a iniquidade, o financiamento insuficiente e a má gestão dos recursos do sistema. Muitos desses problemas, como veremos, podem ter sua raiz nos conceitos trazidos pela Constituição e em elementos hegemônicos do pensamento sanitarista.

Além disso, o desenvolvimento do conhecimento científico e o acúmulo de experiência com os sistemas de saúde ao redor do mundo não podem ser ignorados. Se o movimento da reforma sanitária possibilitou o marco legal para a construção do SUS, parece ter avançado pouco nas questões conceituais que permitiriam tornar de fato a Atenção Primária ordenadora do sistema, ${ }^{2}$ como a discussão da contratualização dos serviços privados, nos modelos de remuneração dos profissionais, nos processos de regulação da clínica, dentre outros aspectos. O processo insular e autorreferenciado em que vive a discussão acadêmica sobre modelos de organização do sistema de saúde pode ser apontado como uma das razões pelas quais não temos sido capazes de debater com profundidade problemas elementares.

Pretende-se, nesse artigo, apresentar e discutir as bases para novas formas de organizar o sistema de saúde e de fortalecer a Atenção Primária à Saúde (APS), nos níveis macro, intermediário e micro.

\section{Nível macro}

A principal referência para a construção de redes de atenção é o relatório Dawson, que descreveu os setores primário, secundário e terciário. Neste documento de $1920^{3}$ está o indicativo que a saúde deve ser regionalizada e não municipalizada. Ou seja, no Brasil o que aconteceu foi uma "descentralização centralizadora", fazendo com que muitas vezes até o Ministério da Saúde atue como um "grande município" ao tratar de questões locais e do nível micro, sendo que os estados que deveriam ter maior protagonismo na construção da rede ou sofrem bypass ou não exercem sua função.

O sistema de saúde é considerado público se o financiamento é majoritariamente público (preferencialmente, ao menos $70 \%$ ), mas os serviços podem ser na sua maioria privados desde que regulados pelo Estado. O conceito de privado inclui “pertencente ao próprio trabalhador". Esta é a realidade na maioria dos países com sistema de saúde universal de fato e financiamento majoritariamente público como Canadá, Japão, Holanda, Inglaterra, Dinamarca, Irlanda, Itália, Noruega, Nova Zelândia, etc... onde 
a rede de serviços de atenção primária à saúde (consultórios) pertence aos próprios médicos que fazem contratos direto com o governo. As exceções são Portugal, Espanha, Suécia e Finlândia onde a força de trabalho é estatal, sendo que em todos esses países há reformas que direcionam para uma maior autonomia do trabalhador regulada por meio de uma complexa contratualização. ${ }^{4}$

No Brasil, ao se criar o SUS em 1988, abre-se mão de uma rede de clínicos gerais que atendiam como profissionais liberais ou contratados pelo Instituto Nacional de Previdência Social (INPS) em todo o país e opta-se pela construção de uma Atenção Primária com provimento público (estrutura física e funcionários públicos). Se na Inglaterra a APS do National Health Service (NHS) origina-se da clínica individual e com o tempo passa a incorporar algumas medidas preventivas (como vacinas) e trabalho multiprofissional, no Brasil a APS no SUS tem origem no posto de vacinação e nas ações programáticas. Esse caminho explica a dificuldade atual de fazer a APS brasileira incorporar o que mundo afora é a sua essência: a clínica dos problemas mais comuns. Outra consequência foi a perda de uma imensa força de trabalho médica que passou a ver o serviço público como secundário. Não terão sido justamente esses clínicos gerais liberais espalhados por todo o país que foram absorvidos pelos planos de saúde para criar a sua rede de serviços? Não teria sido melhor o SUS tê-los absorvido e a partir deles organizar uma APS e atenção especializada contratualizada e regulada pelo Estado?

Os sistemas de saúde bismarckianos e beveridgianos, embora tenham uma diferença original quanto ao financiamento, ou seja, enquanto no bismarckiano há um imposto específico para a saúde e no beveridgiano o sistema é custeado por impostos gerais, eles se convergem desde que o financiamento seja majoritariamente público. Embora os recursos arrecadados nos sistemas bismarckianos sejam geridos por autarquias consideradas privadas, este recurso é regulado pelo Estado e, portanto, é um recurso público. Assim, não há a condenação a priori de um desses sistemas. O que importa é a forma de gerir o dinheiro e como o sistema é regulado em um nível intermediário entre o macro e o micro. A principal vantagem do sistema bismarckiano é que tende a despolitizar a gestão da saúde, enquanto o beveridgiano tende a fortalecer a equidade. As principais desvantagens são os opostos. É preciso avaliar como cada cultura aceita os modelos e como eles são implementados. Na maioria dos países não é o sistema (bismarckiano ou beveridgiano) em si o problema, mas a forma de implantação, o grau de corrupção e de politização ou de mercantilização da saúde. O mais relevante é que o sistema e os serviços de saúde sejam socializados. ${ }^{5,6}$

É importante haver um equilíbrio entre equidade e liberdade, sendo que o Estado deve proteger sempre o primeiro na correlação de forças. Sistemas bismarckianos tendem a favorecer a liberdade, enquanto o beveridgiano a equidade, mas esta tendência pode ser corrigida pela regulação do Estado. Alguns países bismarckianos como a Holanda tomaram medidas neste sentido, com resultados positivos relevantes. $\mathrm{O}$ Brasil passou de um sistema bismarckiano (INPS) para um beveridgiano (SUS), mas manteve resquícios do anterior como os sistemas de autogestão (Cassi e GEAP), Hospitais dos Servidores Estaduais e Municipais, Militares, etc...

Outro problema criado com o SUS em 1988 e reforçado com a NOB 91 e 92 foi a municipalização dos serviços de forma irresponsável. ${ }^{7,8}$ Toda a rede de atenção primária existente foi passada aos municípios, bem como a responsabilidade da sua estruturação, sem dar condições financeiras e de gestão para que o sistema funcionasse a contento. Alguns grandes municípios conseguiram razoavelmente 
se estruturar e atingir um nível de qualidade da APS, mas a grande maioria dos municípios brasileiros mostra uma falta de capacidade na gestão dos serviços e isso não é exclusivo das pequenas cidades, já que existem inúmeros exemplos de metrópoles que se arrastam na estruturação de sua APS com resultados pífios há muitos anos.

Boa parte do problema está ligada à excessiva politização inerente à escolha do modelo beveridgiano, mas soma-se a isso a falta de formação mínima para atuação na área da maioria dos gestores municipais e a insolvência financeira de grande parte dos municípios brasileiros. O mais grave é que essa decisão tomada na década de 90 enfraquece o fator que deveria ser o ponto forte de um sistema beveridgiano, que é a equidade, já que o sistema não é único e sim composto por mais de 5.600 subsistemas fragmentados e com padrões de qualidade diferentes dependendo da cidade em que está implementado, acarretando em uma competição e autofagia. No caso brasileiro, a equidade se dá com sorte dentro da mesma cidade, mas muitas vezes nem isso. De todos os países com sistemas de fato universais de saúde, apenas a Finlândia, um país com pouco mais de cinco milhões de habitantes, tem uma municipalização semelhante, o que demonstra que foi uma ideia desprovida de referências e de muito difícil execução. Já nas décadas de 60 e 70 havia certo acúmulo de experiências quanto à melhor forma de promover a descentralização. Tudo leva a crer que a opção pela municipalização foi uma decisão com viés corporativista e político, pois com a autonomia municipal cada prefeito ou secretario municipal de saúde ganhou uma importância na formulação da política de saúde desproporcional à capacidade de implementação, além de cada nova eleição representar uma ameaça ao que vinha sendo construído. As cidades com mais sucesso são as que seguem a formulação nacional quando esta está de acordo com as premissas beveridgianas sem procurar inventar modelos próprios.

Além disso, no Brasil o sistema privado de inspiração norte-americana responde por mais de $50 \%$ dos gastos e em um nível micro ainda sobrevivem unidades de saúde tradicionais com foco em agendas e ações programáticas com internistas, ginecologistas e pediatras de inspiração soviética. Ou seja, os quatro modelos coexistem no Brasil representados principalmente pela Estratégia Saúde da Família (beveridgiano), autogestão (bismarckiano), Unidades Básicas de Saúde (UBS) chamadas tradicionais (soviético) e medicina de grupo ou planos de saúde (norte-americano). ${ }^{9}$

O conceito de integralidade necessita ser contextualizado. Em cada contexto se utiliza o termo "integralidade" de uma maneira distinta, sendo que para o Sistema Único de Saúde faz alusão às ações de promoção, prevenção, cura e reabilitação. Já quando presente nos atributos da Atenção Primária á Saúde denota "cuidado abrangente" (do inglês comprehensiveness), ou seja, faz referência à carteira de serviços e sua amplitude. Na definição de APS, o conceito de prevenção não é utilizado, mas é intrínseco a praticamente todas as ações na área da saúde. É mais prático pensar em integralidade como toda a oferta de serviços e de cuidados de um sistema de saúde (como o SUS). E em abrangência como um atributo central da Atenção Primária, que não pode dividir sua oferta de serviços em função de uma faixa etária ou de um problema/patologia. A utilização do conceito de integralidade como a abordagem ampliada da pessoa (contexto cultural, familiar e comunitária) representa para Starfield atributos derivados da APS, ou seja, é papel desse espaço de atenção, mas ocorrerá apenas nas equipes/UBS que cumprirem minimamente os atributos nucleares: acesso, continuidade, abrangência dos serviços e coordenação do cuidado. ${ }^{10,11}$ 
É importante resgatar as ideias de Geoffrey Rose para monitorar as intervenções populacionais e individuais, assim como os paradoxos do risco e da prevenção. ${ }^{12}$ As intervenções essencialmente individuais não podem ser utilizadas em um nível populacional e vice-versa. Os serviços de saúde assistenciais em um nível micro têm como principal função a intervenção individual. Um exemplo de confusão de intervenção individual e populacional é a visita mensal dos agentes comunitários de saúde em todas as casas da área de abrangência. Esta ação específica não tem resultados de custo efetividade demonstrados e deveria ser focada nos indivíduos em risco e de maior vulnerabilidade, considerando que quase sempre há heterogeneidade nas diversas populações.

\section{Nível intermediário}

É preciso reavaliar cotidianamente a prática com indicadores de estrutura, processo e resultado com foco nos atributos nucleares da APS. Um dos instrumentos mais bem acabados para fazer esta avaliação é o Primary Care Assessment Tool (PCATool), traduzido e validado para a realidade brasileira. ${ }^{13}$ Outras ferramentas adaptadas às necessidades locais devem ser usadas como internações por condições sensíveis à APS, mortalidade evitável, atraso no diagnóstico, resultados em saúde segundo a classe social e nível de educação formal, resistências bacterianas, etc.

A vigilância epidemiológica não deve ditar a agenda e a gestão da clínica. É louvável que no Brasil haja uma integração da vigilância em saúde com a assistência, em especial nas unidades de saúde. Mas não pode haver sobreposição ou mesmo imposição da agenda da vigilância a todos os profissionais da saúde. O desafio é não perder as conquistas como a ampla cobertura vacinal, mas avançar para uma prática centrada na demanda das pessoas, nos riscos individuais e no acesso. Não basta avaliar as ações programáticas como programas de vacinação e tuberculose para compreender a atenção primária como um todo. Outros instrumentos citados como internações por condições sensíveis à APS devem ser igualmente valorizados. É inaceitável que um idoso seja anualmente vacinado contra influenza mas morra de insuficiência cardíaca por falta de assistência desde a atenção primária. Ao mesmo tempo, é importante rever possíveis exageros em protocolos pouco custo efetivos e com alto custo de oportunidade, valores que não são mensurados, em especial em sistemas muito verticalizados. Problemas de saúde ou faixas etárias que não estão em programas de vigilância muitas vezes são negligenciados. Os responsáveis pelos programas verticais das secretarias municipais, estaduais e mesmo do Ministério da Saúde se beneficiariam se mantivessem uma carga horária parcial na assistência como generalistas para, assim, terem mais condições de adequar os protocolos à realidade, procurando promover resultados globais positivos e não o predomínio de um programa sobre os demais, respeitadas as variações sazonais, endemias e epidemias.

A forma de remuneração em todos os níveis deve ser através de composições (mix), sendo que na atenção primária à saúde o modelo de capitação, onde houver lista de pacientes, deve representar pelo menos $50 \%$ da remuneração global. Nenhuma das formas de remuneração (capitação, salário fixo, produção e resultado) deve corresponder a $100 \%$ da remuneração. ${ }^{14}$ É importante incorporar algum mecanismo que premie o profissional que fique mais tempo na mesma unidade. É igualmente relevante proporcionar incentivos para que o médico e sua família vá para o meio rural com mais recursos materiais, viagens para formação continuada, bolsas de estudo universitárias preferenciais para os filhos destes profissionais, etc. 
Os médicos gerais devem ter listas de pacientes que de preferência não devem seguir regiões geográficas, mas sim listas abertas cuja carga deve ser avaliada por critérios como idade e vulnerabilidade social, além de parâmetros estudados (como adjusted case groups ${ }^{15}$ ou fórmula de Carr-Hill ${ }^{16}$ ) e adequados à realidade local. A área geográfica deve ser da unidade de saúde onde o profissional seria responsável pela visita domiciliar, ou seja, dentro desta área geográfica o cidadão pode escolher seu médico. O ideal é que este mesmo profissional ajude no cuidado fora do horário comercial na forma de rodízio regional.

Deve haver uma política de regulação da formação médica clara que reserve entre 35 e $40 \%$ de todas as vagas de residência para a medicina de família e comunidade como ocorre em grande parte da Europa Ocidental e Canadá. ${ }^{17}$ A residência deve ser mandatória para se praticar medicina sem supervisão e deve ser entendida como um mecanismo de regulação do mercado, além de uma estratégia bem acabada e custo efetiva de formação em serviço.

\section{Nível micro}

O acesso deve ser prioritariamente com médicos generalistas bem formados. No Brasil, não há uma definição clara e única de "generalista", mas o conjunto de conhecimentos mais bem definido e adequado para receber o paciente no sistema de saúde e fazer o primeiro filtro aumentando a probabilidade pré-teste para possíveis intervenções dos especialistas é a medicina de família e comunidade. ${ }^{18} \mathrm{O}$ pediatra é o especialista mais próximo do médico de família e comunidade, mas pode ser mais bem aproveitado em outros cenários. A coordenação dos cuidados deve ser realizada pelo médico generalista, sendo que os especialistas focais devem prestar serviços temporários e de assessoria.

O acesso do paciente na atenção primária é prioritário. As agendas nas unidades de saúde ou consultórios de médicos gerais não devem ser programáticas, ou seja, não deve haver dia ou período para hipertensão, diabetes, idoso ou saúde mental. O risco deve ser individualizado e não de acordo com "programas". Ou seja, um diabético compensado pode não ser visto no mesmo dia, enquanto um paciente com sangramento digestivo esporádico precisa ser atendido imediatamente. Os conceitos de acesso avançado e agendamento não devem ser dicotomizados, ou seja, o agendamento deve ser usado para favorecer o acesso e não para criar uma fila virtual. Caso o acesso esteja comprometido, é preciso avaliar quantitativamente as variáveis implicadas como tempo de consulta, pressão assistencial, lista de pacientes e "frequentação". ${ }^{19}$

Os conceitos de prevenção quaternária ${ }^{20}$ e de sobrediagnóstico ${ }^{21}$ devem ser incorporados no cotidiano da APS de forma qualificada, integrando-se com outras tecnologias como a medicina baseada em evidência. É fundamental que a implementação desses conceitos seja monitorada com indicadores provenientes do PCATool, das internações por condições sensíveis a APS, ${ }^{22}$ dentre outros instrumentos.

Devem ser incorporadas tecnologias leves (soft) ${ }^{23}$ que demonstraram benefícios aos pacientes e ao sistema como o "método clínico centrado na pessoa". ${ }^{24} \mathrm{O}$ profissional da saúde tem poder iatrogênico como qualquer exame e, portanto, suas tecnologias devem ser testadas para serem incorporadas. Uma ideia não pode ser considerada adequada apenas por ter uma base filosófica sólida. É preciso fortalecer os atributos da Atenção Primária à Saúde (acesso, coordenação, longitudinalidade e cuidado abrangente) e colaborar efetivamente na clinica do médico geral. 
Faltam estudos que demonstrem a efetividade e o benefício ao sistema dos conceitos de acolhimento, ${ }^{25}$ projeto terapêutico singular, ${ }^{26}$ matriciamento ${ }^{27}$ e clínica ampliada. ${ }^{28}$ É importante avaliar como cada uma dessas definições utilizadas no nível micro alteram os atributos essenciais da APS em um nível intermediário. O acesso, o cuidado colaborativo, a discussão de caso, a individualização da conduta com empoderamento das pessoas, as habilidades de comunicação são campos de estudo amplos e fazem parte da base do trabalho na APS, sendo que a ressignificação desses conceitos-chave precisa ser acompanhada de demonstração de efetividade.

O sistema de registro deve seguir a sistematização SOAP e o Registro Clínico Orientado por Problemas como descrito por Lawrence Weed. ${ }^{29}$ A melhor forma de classificação na Atenção Primária à Saúde é por meio da Classificação Internacional de Atenção Primária (CIAP). ${ }^{30}$ Todo prontuário na atenção primária deve ter uma Lista de Problemas bem sistematizada.

\section{Dicotomias}

Ao longo dos anos, algumas dicotomias no Brasil foram sendo acentuadas o que, no cotidiano, gera confusão para a população e fazem os serviços ficarem sobrepostos e pouco custo efetivos. As principais dicotomias são:

Público versus privado: como já explicitado, o financiamento deve ser majoritariamente público e o serviço pode ser majoritariamente privado, desde que regulado por meio, principalmente, da compra de grande quantidade de serviços e da adequada contratualização (via de regra, nos países onde o sistema de saúde foi bem sucedido este foi o caminho). O que chama a atenção é que este processo de "integração" foi acontecendo sem uma coordenação do Estado, resultando nas Organizações Sociais como prestadoras dos serviços para o sistema público, ao invés de empoderar os trabalhadores fazendo contratos diretos com suas respectivas pessoas jurídicos como é comum na Europa. Mesmo assim, aceita-se a contratualização para serviços hospitalares, mas há uma resistência corporativista de sindicatos e movimentos vinculados ao antigo sanitarismo com relação aos serviços privados na atenção primária. Muitos sindicatos exigem que os trabalhadores sejam estatutários e ao mesmo tempo reivindicam que o empregador, muitas vezes o município ou uma instituição pública como a Fiocruz, subsidie planos de saúde privados, o que representa uma contradição com potencial de ameaçar o futuro do sistema de saúde brasileiro.

Salário fixo versus produção: estas são as duas formas de remuneração mais usadas. O salário é hegemônico no sistema público, enquanto o pagamento por produção ainda predomina no sistema privado. Muitas vezes, estes componentes representam $100 \%$ da remuneração pelo serviço. O mix deveria estar no mesmo empregador representado em cada paciente ou consulta, mas na realidade o médico faz esta composição de maneira artificial com diferentes vínculos, um pagando fixo e outro por produção.

Agudo versus crônico: esta dicotomia muitas vezes é retomada ciclicamente e tem origem na fisiopatologia e na determinação arbitrária de patologias "agudas" e "crônicas". Por exemplo, sinusite até um certo período de dias é considerada aguda e depois é considerada crônica. Para o paciente, o que mais importa é o sofrimento, as limitações na função, a expectativa com relação ao sistema de saúde, etc. Por isso, na atenção primária não cabem escalas como a de Manchester, que priorizam sinais e sintomas 
"físicos" (embora não seja possível fazer uma distinção clara entre físico e mental, foi usado aqui um termo corriqueiro exclusivamente para a compreensão da ideia). Uma unidade de emergência pode referenciar pacientes de baixo risco ou "crônicos", mas uma unidade de atenção primária já é o primeiro contato tanto para casos agudos quanto crônicos. Independentemente do problema, na atenção primária as pessoas são cuidadas longitudinalmente, sejam os problemas crônicos, agudos ou crônicos-agudizados. Uma pessoa sem doenças mas com "medo de estar com um problema grave" por qualquer motivo deve ser atendida o mais rápido possível. Ou seja, na atenção primária deve-se promover a integração "corpo" e "mente" e qualquer demanda de qualquer pessoa é relevante. ${ }^{31}$ A dicotomia "agudo" versus "crônico" representa uma ameaça a esta premissa.

\section{Conclusão}

Provavelmente, há grande influência da Constituição de 1988 na agudização dessas dicotomias e contradições. O artigo 196 diz que a "A saúde é direito de todos e dever do Estado" e assim lança as bases do Sistema Único de Saúde (SUS), enquanto o artigo 199 estabelece que "a saúde é livre à iniciativa privada". Ou seja, trata-se de uma constituição que previa o que aconteceria ou determinou a realidade atual. Estes artigos foram frutos da mobilização e lobby de cada uma das principais forças vigentes até 1988: o Movimento da Reforma Sanitária de um lado e os planos de saúde privados, medicina de grupo e cooperativas médicas de outro. Cada um construiu o "seu" sistema de saúde. Este sanitarismo teve grande importância até a Constituição de 1988. Embora fosse desconectado dos princípios que norteavam os principais sistemas de saúde do mundo, como a integração do sistema com financiamento público com o serviço privado, liderados pelo movimento inglês que já adotava estas medidas desde a década de 50, e de ser irrealisticamente estatizante, evitou que o sistema de saúde brasileiro fosse americanizado ou mercantilizado por completo. Porém, pode-se dizer que o artigo 199 representa na verdade a derrota do Movimento da Reforma Sanitária, cuja resultante foi o financiamento majoritariamente privado vigente.

Ao mesmo tempo, o artigo 196 faz alusão ao conceito de "saúde como um direito", sendo que a própria definição de "saúde" é motivo de intermináveis debates. O professor Juan Gérvas, que inspirou grande parte dessas ideias aqui descritas, ${ }^{32,33}$ sempre que veio ao Brasil deixou claro que não se pode prometer o intangível como a felicidade. ${ }^{34}$ Ou seja, o máximo que deve estar descrito na Constituição é que serviços de saúde custo efetivos são um direito da população, e já há parâmetros que precisam ser debatidos para definir o que é custo efetivo para a realidade brasileira.

É preciso fazer a reforma e esta deve começar da base. Modificar a Constituição e estes dois artigos extremamente contraditórios é apenas o primeiro passo. Este texto pretende dar um norte e propor princípios para os passos seguintes. Nas décadas de 60 e 70, os movimentos eram muito fechados e faltou comparar sistemas de saúde. Hoje, há mais informações e não se pode viver de crenças e ideologias que ficaram no passado e que paradoxalmente alimentam o perverso e o oposto. Atualmente, a população chegou a uma situação limite no Brasil em que o principal incentivador do serviço público é o privado e do serviço privado é o público. A solução não é financiar os dois. 


\section{Referências}

1. Teixeira SF, org. Reforma Sanitária: em busca de uma teoria. São Paulo: Cortez/Abrasco; 1989.

2. Starfield B. Primary care: an increasingly important contributor to effectiveness, equity, and efficiency of health services. SESPAS report 2012. Gac Sanit. 2012;26 Suppl 1:20-6. DOI: http://dx.doi.org/10.1016/j.gaceta.2011.10.009

3. Dawson B. Informe Dawson sobre el futuro de los serviços médicos y afines, 1920. Washington: Organización Panamericana de la Salud, Publicacion Científica no 93;1964.

4. Gérvas J. Perez Fernandez M. Organização da Atenção Primária à Saúde em Outros Países. In: Gusso G, Lopes JM. Tratado de Medicina de Família e Comunidade: Princípios, Formação e Prática. ArtMed: Porto Alegre; 2012.

5. Gérvas J, Pérez Fernández M, Starfield BH. Primary care, financing and gatekeeping in western Europe. Fam Pract. 1994;11(3):307-17. DOI: http://dx.doi.org/10.1093/fampra/11.3.307

6. Giovanella L. A atenção primária à saúde nos países da União Européia: configurações e reformas organizacionais na década de 1990. Cad Saúde Pública. 2006;22(5):951-63. DOI: http://dx.doi.org/10.1590/S0102-311X2006000500008

7. Brasil. Constituição (1988). Constituição da República Federativa do Brasil. São Paulo: Revista dos Tribunais; 1997.

8. Brasil. Ministério da Saúde. Portaria no 234, de 07 de fevereiro de 1992. Edição da Norma Operacional Básica do Sistema Único de Saúde para 1992 (NOB-SUS/92). Brasília: Ministério da Saúde; 1992.

9. Roemer MI. National Strategies for Health Care Organization: a World Overview. Ann Arbor: Health Administration Press; 1985.

10. Starfield B. Atenção primária: equilíbrio entre necessidades de saúde, serviços e tecnologia. Brasília: UNESCO/Ministério da Saúde; 2002.

11. Starfield B, Hyde J, Gérvas J, Heath I. The concept of prevention: a good idea gone astray? J Epidemiol Community Health. 2008;62(7):580-3. DOI: http://dx.doi.org/10.1136/jech.2007.071027

12. Rose G. Estratégias da medicina preventiva. Porto Alegre: Artmed; 2010. 192p.

13. Brasil. Ministério da Saúde. Secretaria de Atenção em Saúde. Departamento de Atenção Básica. Manual do instrumento de avaliação da atenção primária à saúde. Primary Care Assessment Tool PCATool-Brasil. Ministério da Saúde, Secretaria de Atenção em Saúde, Departamento de Atenção Básica. Brasília: Ministério da Saúde; 2010.

14. An Optimal remuneration system for General Practitioners. [homepage na Internet] Nederlandse Zorgautoriteit. [Acesso 27 Jul 2014]. Disponível em: http://www.nza.nl/104107/230942/Paper_-_An_Optimal_remunera1.pdf

15. Starfield B, Kinder K. Multimorbidade e sua mensuração. In: Gusso G, Lopes JM. Tratado de Medicina de Família e Comunidade: Princípios, Formação e Prática. Porto Alegre: ArtMed; 2012.

16. Review of the General Medical Services - global sum formula [homepage na Internet] National Health System [Acesso 27 Jul 2014]. Disponível em: http://www.nhsemployers.org/ /media/Employers/Documents/Primary\%20care\%20contracts/ GMS/GMS\%20Finance/Global\%20Sum/frg_report_final_cd_090207.pdf

17. CARMS [home page na Internet]. Canadian Resident Matching Service. [Acesso 9 Abr 2014]. Disponível em: https://www. carms.cal

18. McWhinney IR, Freeman T. Manual de medicina de família e comunidade. 3a ed. Porto Alegre: ArtMed; 2010.

19. Gusso G, Poli Neto P. Gestão da Clínica. In: Gusso G, Lopes JMC. Tratado de medicina de família e comunidade: princípios, formação e prática. ArtMed: Porto Alegre; 2012. p.159-66.

20. Jamoulle M, Gusso G. Prevenção quaternária: primeiro não causar dano. In: Gusso G, Lopes JMC, orgs. Tratado de medicina de família e comunidade: princípios, formação e prática. ArtMed: Porto Alegre; 2012. p. 2015-211.

21. Welch HG, Schwartz L, Woloshin S. Overdiagnosed: making people sick in the pursuit of health. Boston: Beacon Press; 2011.

22. Alfradique ME, Bonolo Pde F, Dourado I, Lima-Costa MF, Macinko J, Mendonça CS, et al. Ambulatory care sensitive hospitalizations: elaboration of Brazilian list as a tool for measuring health system performance (Project ICSAP--Brazil). Cad Saude Pública. 2009;25(6):1337-49. DOI: http://dx.doi.org/10.1590/S0102-311X2009000600016 
23. Feinstein AR. The need for humanised science in evaluating medication. Lancet. 1972;2(7774):421-3. DOI: http://dx.doi. org/10.1016/S0140-6736(72)91814-4

24. Stewart M, Brown JB, Weston WW, McWhinney IR, McWilliam CL, Freeman TR. Patient centered medicine: transforming the clinical method. London: Radcliffe Publishing; 2014.

25. Franco TB, Bueno WS, Merhy EE. O acolhimento e os processos de trabalho em saúde: o caso de Betim, Minas Gerais, Brasil. Cad Saúde Pública. 1999;15(2):345-53. DOI: http://dx.doi.org/10.1590/S0102-311X1999000200019

26. Oliveira GN. O projeto terapêutico como contribuição para a mudança das práticas de saúde [Dissertação de mestrado]. Campinas: Faculdade de Ciências Médicas, Universidade Estadual de Campinas; 2007.

27. Campos GWS. Equipes de referência e apoio especializado matricial: um ensaio sobre a reorganização do trabalho em saúde. Ciênc Saúde Coletiva. 1999;4(2):393-403. DOI: http://dx.doi.org/10.1590/S1413-81231999000200013

28. Cunha GT. A construção da Clínica ampliada na atenção básica. São Paulo: Hucitec; 2005. p.212.

29. Weed LL. Medical records that guide and teach. N Engl J Med. 1968;278(11):593-600. PMID: 5637758 DOI: http://dx.doi. org/10.1056/NEJM196803142781105

30. World Organization of National Colleges, Academies, and Academic Associations of General Practitioners/Family Physicians. Classificação Internacional de Atenção Primária (CIAP 2). $2^{2}$ ed. Florianópolis: Sociedade Brasileira de Medicina de Família e Comunidade; 2009.

31. Damásio A. Em busca de Espinosa: prazer e dor na ciência dos sentimentos. São Paulo: Companhia das Letras;2004. p.358.

32. Gérvas J, Pérez Fernandez M. Uma atenção primária forte no Brasil. Relatório sobre como fortalecer os acertos e corrigir as fragilidades da estratégia saúde da família. [acesso em 11 jan 2012]. Disponível em: www.sbmfc.org.br/media/file/ documentos/relatoriofinal_portugues.pd

33. Casajuana J, Gérvas J (directores). La renovación de la atención primaria desde la consulta. Madrid: Springer-Health Care Ibérica; 2012.

34. Gérvas J. Atención Primaria, de la teoría a la práctica. Cad Saúde Pública. 2008;24 Sup1:S24-S26. 